\title{
EXACT CONTROLLABILITY TO THE TRAJECTORIES OF THE HEAT EQUATION WITH FOURIER BOUNDARY CONDITIONS: THE SEMILINEAR CASE*
}

\author{
Enrique Fernández-Cara ${ }^{1}$, Manuel González-Burgos ${ }^{1}$, Sergio Guerrero ${ }^{2}$ \\ AND JEAN-PIERRE PUEL ${ }^{3}$
}

\begin{abstract}
This paper is concerned with the global exact controllability of the semilinear heat equation (with nonlinear terms involving the state and the gradient) completed with boundary conditions of the form $\frac{\partial y}{\partial n}+f(y)=0$. We consider distributed controls, with support in a small set. The null controllability of similar linear systems has been analyzed in a previous first part of this work. In this second part we show that, when the nonlinear terms are locally Lipschitz-continuous and slightly superlinear, one has exact controllability to the trajectories.
\end{abstract}

Mathematics Subject Classification. 35K20, 93B05.

Received February 17, 2005. Revised May 30, 2005 and June 13, 2005.

\section{IntroduCtion}

Let $\Omega \subset \mathbf{R}^{N}(N \geq 1)$ be a bounded connected open set whose boundary $\partial \Omega$ is regular enough (for instance $\partial \Omega \in C^{2}$ ). Let $\omega \subset \Omega$ be a (small) nonempty open subset and let $T>0$. We will use the notation $Q=\Omega \times(0, T)$ and $\Sigma=\partial \Omega \times(0, T)$ and we will denote by $n(x)$ the outward unit normal to $\Omega$ at the point $x \in \partial \Omega$.

We will consider the semilinear heat equation with nonlinear Fourier (or Robin) boundary conditions

$$
\begin{cases}y_{t}-\Delta y+F(y, \nabla y)=v 1_{\omega} & \text { in } Q, \\ \frac{\partial y}{\partial n}+f(y)=0 & \text { on } \Sigma, \\ y(x, 0)=y^{0}(x) & \text { in } \Omega .\end{cases}
$$

Here, we assume that $v \in L^{2}(\omega \times(0, T))$ (at least), $1_{\omega}$ is the characteristic function of $\omega, y^{0} \in L^{\infty}(\Omega)$ and $F: \mathbf{R} \times \mathbf{R}^{N} \mapsto \mathbf{R}$ and $f: \mathbf{R} \mapsto \mathbf{R}$ are given functions. In $(1), y=y(x, t)$ is the state and $v=v(x, t)$ is the control; it is assumed that we can act on the system only through $\omega \times(0, T)$.

\footnotetext{
Keywords and phrases. Controllability, heat equation, Fourier boundary conditions, semilinear.

* This work has been partially supported by D.G.E.S. (Spain), Grants BFM2000-1317 and BFM2003-06446.

1 Dpto. E.D.A.N., University of Sevilla, Aptdo. 1160, 41080 Sevilla, Spain; cara@us.es; manoloburgos@us.es; sguerrero@us.es

2 Laboratoire Jacques-Louis Lions, Université Pierre et Marie Curie, boîte courrier 187, 75035 Cedex 05, Paris, France; guerrero@ann.jussieu.fr

${ }^{3}$ Laboratoire de Mathématiques Appliquées, Université de Versailles - St. Quentin, 45 avenue des États-Unis, 78035 Versailles, France; jppuel@cmapx.polytechnique.fr

(C) EDP Sciences, SMAI 2006
} 
For the existence, uniqueness, regularity and general properties of the solutions to problems like (1), see for instance $[1,2,7]$. An illustrative interpretation of the data and variables in (1) is the following. The function $y=y(x, t)$ can be viewed as the relative temperature of a medium (with respect to the exterior surrounding air) subject to transport and chemical reactions. The parabolic equation in (1) means, among other things, that a heat source $v 1_{\omega}$ is applied on a part of the body. On the boundary, $-\frac{\partial y}{\partial n}$ can be viewed as the normal heat flux, inwards directed, up to a positive coefficient. Thus, the equality

$$
-\frac{\partial y}{\partial n}=f(y)
$$

means that this flux is a (nonlinear) function of the temperature. Accordingly, it is reasonable to assume that $f$ is nondecreasing and $f(0)=0$.

A simplified linear model which was considered in a previous paper [10] is the following:

$$
\begin{cases}y_{t}-\Delta y+a(x, t) y+B(x, t) \cdot \nabla y=v 1_{\omega} & \text { in } Q \\ \frac{\partial y}{\partial n}+\beta(x, t) y=0 & \text { on } \Sigma \\ y(x, 0)=y^{0}(x) & \text { in } \Omega .\end{cases}
$$

Here, it is assumed that the coefficients $a, B$ and $\beta$ satisfy

$$
a \in L^{\infty}(Q), \quad B \in L^{\infty}(Q)^{N}, \quad \beta \in L^{\infty}(\Sigma)
$$

and, for the reasons above, it is also natural to assume that $\beta \geq 0$ (although this assumption was not used in $[10])$.

The main goal of this paper is to analyze the controllability properties of the nonlinear system (1). More precisely, we will try to reach exactly uncontrolled solutions of $(1)$, i.e. functions $\bar{y}=\bar{y}(x, t)$ satisfying

$$
\begin{cases}\bar{y}_{t}-\Delta \bar{y}+F(\bar{y}, \nabla \bar{y})=0 & \text { in } Q, \\ \frac{\partial \bar{y}}{\partial n}+f(\bar{y})=0 & \text { on } \Sigma \\ \bar{y}(x, 0)=\bar{y}^{0}(x) & \text { in } \Omega .\end{cases}
$$

It will be said that (1) is (globally) exactly controllable to the trajectories at time $T$ if, for any solution of (4) with "suitable" regularity and any $y^{0} \in L^{\infty}(\Omega)$, there exist controls $v \in L^{2}(\omega \times(0, T))$ and associated solutions $y \in C^{0}\left([0, T] ; L^{2}(\Omega)\right)$ such that

$$
y(x, T)=\bar{y}(x, T) \text { in } \Omega .
$$

Here, by suitable regularity we mean the following:

$$
\bar{y} \in L^{2}\left(0, T ; H^{1}(\Omega)\right) \cap C^{0}\left([0, T] ; L^{2}(\Omega)\right) \cap L^{\infty}(Q), \quad \bar{y}^{0} \in L^{\infty}(\Omega) .
$$

The controllability properties of semilinear time-dependent systems have been studied intensively these last years. See for instance $[8,11-13,15,16]$, where nonlinearities of the form $f(y)$ are considered. See also the general treatise [14]. In particular, for parabolic systems completed with Dirichlet boundary conditions, nonlinear terms $f(y, \nabla y)$ depending on both the state and the gradient have been taken into account in [6,9]. For the similar linear system (2), the null controllability was analyzed more in detail in [10]. In the case of (1), some partial results have been given in [5]. 
Our main result concerns the global exact controllability to the trajectories of (1). It is the following:

Theorem 1. Let us assume that $F$ and $f$ are locally Lipschitz-continuous and satisfy

$$
\lim _{|s| \rightarrow \infty} \frac{|F(s, p)-F(r, p)|}{|s-r| \log ^{3 / 2}(1+|s-r|)}=0,
$$

uniformly in $(r, p) \in[-K, K] \times \mathbf{R}^{N} \forall K>0$,

$$
\left\{\begin{array}{l}
\forall L>0, \exists M>0 \text { such that } \\
|F(s, p)-F(r, p)| \leq M|s-r|, \quad|F(s, p)-F(s, q)| \leq M|p-q| \\
\forall(s, r, p, q) \in[-L, L]^{2} \times \mathbf{R}^{N} \times \mathbf{R}^{N}
\end{array}\right.
$$

and

$$
\lim _{|s| \rightarrow \infty} \frac{|f(s)-f(r)|}{|s-r| \log ^{1 / 2}(1+|s-r|)}=0
$$

uniformly in $r \in[-K, K] \forall K>0$. Then, for each $T>0$, the nonlinear system (1) is exactly controllable to the trajectories at time $T$ with $L^{\infty}$ controls.

Remark 1. Conditions (7)-(9) are satisfied if $F$ and $f$ are globally Lipschitz continuous. Notice that (7) means that the function $F$ can only be slightly superlinear in $s$, uniformly in $p$. In the similar case of Dirichlet boundary conditions, it is known that conditions like these are sharp. Indeed, for instance, when $F$ does not depend on $p$ and

$$
|F(s)-F(r)| \sim|s-r| \log ^{\beta}(1+|s-r|), \quad \beta>2,
$$

due to blow-up phenomena, the system fails to be controllable whenever $\omega \neq \Omega$ (see [11]). On the other hand, (9) is also a slightly superlinear growth assumption for $f$. It would be interesting to know whether a more superlinear $f$ leading to blow up in the absence of control can also be an obstruction for the null controllability of (1). But this question does not seem obvious and remains open.

Remark 2. A result proved in [5] says that when $F \equiv 0, f$ is smooth near zero and

$$
f(s) s \geq 0 \quad \forall s \in \mathbf{R},
$$

the nonlinear system (1) is null controllable for large $T$. That is to say, under these assumptions, for each $y^{0} \in L^{2}(\Omega)$ there exist $T\left(y^{0}\right)>0$ and controls $v$ in $L^{\infty}(\omega \times(0, T)$ such that the associated states $y$ satisfy

$$
y\left(x, T\left(y^{0}\right)\right)=0 \text { in } \Omega .
$$

By inspection of the proof of theorem 1, we see that the same result holds for (1) with $F \equiv 0$ whenever $f$ is locally Lipschitz-continuous and satisfies the good sign condition (10).

For the proof of Theorem 1, we will first establish a null controllability result for (2) (see Prop. 1 below). This will be used, together with an appropriate fixed point argument, to deduce the desired result.

This strategy was introduced in [15] in the framework of the exact controllability of the semilinear wave equation. See also $[8,12]$ for similar results concerning the approximate and null controllability of the semilinear heat equation with Dirichlet or Neumann boundary conditions.

Our null controllability result for (2) is the following:

Proposition 1. For every $T>0$, system (2) is null controllable at time $T$, with controls in $L^{\infty}(\omega \times(0, T))$. More precisely, for each $y^{0} \in L^{2}(\Omega)$, there exists $v \in L^{\infty}(\omega \times(0, T))$ such that the associated solution to (2) satisfies (5). Furthermore, the control $v$ can be found satisfying

$$
\|v\|_{L^{\infty}(\omega \times(0, T))} \leq \mathrm{e}^{C(\Omega, \omega) K\left(T,\|a\|_{\infty},\|B\|_{\infty},\|\beta\|_{\infty}\right)}\left\|y^{0}\right\|_{L^{2}(\Omega)},
$$


where

$$
K=1+1 / T+\|a\|_{\infty}^{2 / 3}+\|B\|_{\infty}^{2}+\|\beta\|_{\infty}^{2}+T\left(1+\|a\|_{\infty}+\|B\|_{\infty}^{2}+\|\beta\|_{\infty}^{2}\right) .
$$

For the proof of proposition 1, we first introduce a control $L^{2}(\omega \times(0, T))$ which leads the solution of (2) to zero at time $T$. In a second step, arguing as in Section 2 in [4], a regularizing argument will lead to the desired $L^{\infty}$ control.

The rest of this paper is organized as follows. In Section 2, we prove Proposition 1. Section 3 is devoted to the proof of Theorem 1. For completeness, we have also included an Appendix where the proof of a rather technical local regularity result is given in detail.

In the sequel, $C$ denotes a generic positive constant only depending on $\Omega$ and $\omega$.

\section{A NUlL CONTROLlability RESUlt FOR THE LiNEAR SYSTEM}

In this section we present the proof of Proposition 1.

Let $y^{0} \in L^{2}(\Omega)$ be given and let us introduce two open sets $\omega^{\prime}$ and $\omega^{\prime \prime}$, with $\omega^{\prime \prime} \subset \subset \omega^{\prime} \subset \subset \omega$. Then, we can use the main result in [10] (Th. 2) with control region $\omega^{\prime \prime} \times(0, T)$ to deduce the existence of a control $\widetilde{v} \in L^{2}\left(\omega^{\prime \prime} \times(0, T)\right)$ such that the associated solution to $(2)$ verifies (5) and also the estimate

$$
\|\widetilde{v}\|_{L^{2}\left(\omega^{\prime \prime} \times(0, T)\right)} \leq \mathrm{e}^{C(\Omega, \omega) K\left(T,\|a\|_{\infty},\|B\|_{\infty},\|\beta\|_{\infty}\right)}\left\|y^{0}\right\|_{L^{2}(\Omega)},
$$

where $K$ is of the form (12).

Let us denote by $\widetilde{y}$ the state associated to $\widetilde{v}$. We now introduce a cut-off function $\eta=\eta(t)$ satisfying

$$
\eta \in C^{\infty}([0, T]), \quad \eta(t)=1 \text { in }(0, T / 4), \quad \eta(t)=0 \text { in }(3 T / 4, T)
$$

and

and we denote by $\chi$ the solution to the system

$$
0 \leq \eta(t) \leq 1,\left|\eta^{\prime}(t)\right| \leq \frac{C}{t} \text { in }(0, T)
$$

$$
\begin{cases}\chi_{t}-\Delta \chi+a(x, t) \chi+B(x, t) \cdot \nabla \chi=0 & \text { in } Q, \\ \frac{\partial \chi}{\partial n}+\beta(x, t) \chi=0 & \text { on } \Sigma, \\ \chi(x, 0)=y^{0}(x) & \text { in } \Omega .\end{cases}
$$

Then, the function $\widetilde{w}=\widetilde{y}-\eta \chi$ satisfies

$$
\begin{cases}\widetilde{w}_{t}-\Delta \widetilde{w}+a(x, t) \widetilde{w}+B(x, t) \cdot \nabla \widetilde{w}=-\eta^{\prime}(t) \chi+\widetilde{v} 1_{\omega^{\prime \prime}} & \text { in } Q, \\ \frac{\partial \widetilde{w}}{\partial n}+\beta(x, t) \widetilde{w}=0 & \text { on } \Sigma, \\ \widetilde{w}(x, 0)=0, \quad \widetilde{w}(x, T)=0 & \text { in } \Omega .\end{cases}
$$

Our aim is to construct a control $v \in L^{\infty}(\omega \times(0, T))$ which drives the solution of (2) to zero at time $t=T$. To this end, we will need a local regularity result for the solutions to linear heat equations with $L^{\infty}$ coefficients $a$ and $B$. This will be used below for the functions $\chi$ and $\widetilde{w}$ and reads as follows:

Lemma 1. Let us denote by $Y$ the space $L^{2}\left(0, T ; H^{1}(\Omega)\right) \cap C^{0}\left([0, T] ; L^{2}(\Omega)\right)$. Let $y \in Y$ be a solution to the equation

$$
y_{t}-\Delta y+a(x, t) y+B(x, t) \cdot \nabla y=f
$$

where $a \in L^{\infty}(Q), B \in L^{\infty}(Q)^{N}$ and $f \in L^{2}(Q)$. Let $\mathcal{O} \subset \Omega$ be a nonempty open set and assume that $f$ is $L^{\infty}$ in the cylinder $\mathcal{O} \times(0, T)$. Then

$$
y \in L^{\infty}\left(\delta, T ; W^{1, \infty}\left(\mathcal{O}^{\prime}\right)\right)
$$


for any $\delta \in(0, T)$ and any nonempty open set $\mathcal{O}^{\prime} \subset \subset \mathcal{O}$. Furthermore, there exists a positive constant $C\left(\mathcal{O}^{\prime}\right)$ such that the following estimate holds:

$$
\|y\|_{L^{\infty}\left(\delta, T ; W^{1, \infty}\left(\mathcal{O}^{\prime}\right)\right)} \leq C\left(\mathcal{O}^{\prime}\right)\left(T^{1 / 2}+T^{N / 2}\right)\left(1+\delta^{-1}+\|a\|_{\infty}+\|B\|_{\infty}\right)^{N+1}\left(\|y\|_{Y}+\|f\|_{L^{\infty}(\mathcal{O} \times(0, T))}\right) .
$$

The previous regularity also holds with $\delta=0$ if, besides (14), we have $y(x, 0)=0$ in $\Omega$. In that case, one has an estimate similar to (15) without the term in $\delta$.

This lemma is implied by well known parabolic regularity theory. For completeness, its proof is given in an Appendix, at the end of this paper.

Let us now consider an open set $\omega_{0}$ with $\omega^{\prime} \subset \subset \omega_{0} \subset \subset \omega$ and a cut-off function $\xi$, with

$$
\xi \in C_{0}^{2}\left(\omega_{0}\right), \quad \xi \equiv 1 \text { in } \omega^{\prime}
$$

and let us set $w=(1-\xi) \widetilde{w}$. Then we have:

$$
\begin{cases}w_{t}-\Delta w+a(x, t) w+B(x, t) \cdot \nabla w=-\eta^{\prime}(t) \chi+v 1_{\omega} & \text { in } Q, \\ \frac{\partial w}{\partial n}+\beta(x, t) w=0 & \text { on } \Sigma, \\ w(x, 0)=0, \quad w(x, T)=0 & \text { in } \Omega,\end{cases}
$$

with

$$
v=\eta^{\prime} \xi \chi+2 \nabla \xi \cdot \nabla \widetilde{w}+\Delta \xi \widetilde{w}-B \cdot \nabla \xi \widetilde{w} .
$$

Let us remark that supp $v \subset \omega \times[0, T]$. Therefore, if we prove that $v \in L^{\infty}(\omega \times(0, T))$, we will have that the function $y=w+\eta \chi$ solves (together with $v$ ) the null controllability problem for (2).

Thus, let us check that $v \in L^{\infty}(\omega \times(0, T))$ and let us estimate its norm in this space:

- The regularity of the first term in the right hand side of (16) is implied by the interior regularity of $\chi$ not only in space but in time as well. From Lemma 1 with $\mathcal{O}=\omega$, we deduce that $\chi \in L^{\infty}\left(\omega_{0} \times(\delta, T)\right)$ with supp $\xi \subset \omega_{0} \subset \subset \omega$ (we even have $\left.\chi \in L^{\infty}\left(\delta, T ; W_{\text {loc }}^{1, \infty}(\omega)\right)\right)$ and

$$
\|\chi\|_{L^{\infty}\left(\omega_{0} \times(\delta, T)\right)} \leq C\left(T^{1 / 2}+T^{N / 2}\right)\left(1+\delta^{-1}+\|a\|_{\infty}+\|B\|_{\infty}\right)^{N+1}\|\chi\|_{Y} ;
$$

recall that $Y=L^{2}\left(0, T ; H^{1}(\Omega)\right) \cap C^{0}\left([0, T] ; L^{2}(\Omega)\right)$.

Consequently taking for instance $\delta=T / 8$, since $\eta^{\prime} \equiv 0$ in $(0, T / 4)$, we get

$$
\left\|\eta^{\prime} \xi \chi\right\|_{L^{\infty}(\omega \times(0, T))} \leq C T^{-1}\left(T^{1 / 2}+T^{N / 2}\right)\left(1+T^{-1}+\|a\|_{\infty}+\|B\|_{\infty}\right)^{N+1}\|\chi\|_{Y} .
$$

- The regularity of the other three terms in the right hand side of (16) is related to the interior space regularity of $\widetilde{w}$. Thus, let us introduce $\omega_{1}$ with $\omega_{0} \subset \subset \omega_{1} \subset \subset \omega$ and let us apply Lemma 1 with $\mathcal{O}=\omega_{1} \backslash \overline{\omega^{\prime \prime}}$. This gives $\widetilde{w} \in L^{\infty}\left(0, T ; W^{1, \infty}\left(\omega_{0} \backslash \overline{\omega^{\prime}}\right)\right)$ and the estimate

$$
\|\widetilde{w}\|_{L^{\infty}\left(0, T ; W^{1, \infty}\left(\omega_{0} \backslash \overline{\omega^{\prime}}\right)\right)} \leq C\left(T^{1 / 2}+T^{N / 2}\right)\left(1+\|a\|_{\infty}+\|B\|_{\infty}\right)^{N+1}\left(\|\widetilde{w}\|_{Y}+\left\|\eta^{\prime} \chi\right\|_{L^{\infty}\left(\omega_{1} \times(0, T)\right)}\right),
$$

whence

$$
\left\{\begin{aligned}
\| 2 \nabla \xi \cdot \nabla \widetilde{w}+\Delta \xi \widetilde{w} & -B \cdot \nabla \xi \widetilde{w} \|_{L^{\infty}(\omega \times(0, T))} \leq C\left(T^{1 / 2}+T^{N / 2}\right) \\
& \times\left(1+\|a\|_{\infty}+\|B\|_{\infty}\right)^{N+2}\left(\|\widetilde{w}\|_{Y}+\left\|\eta^{\prime} \chi\right\|_{L^{\infty}\left(\omega_{1} \times(0, T)\right)}\right) .
\end{aligned}\right.
$$


Putting the previous estimates together, we find that $v \in L^{\infty}(\omega \times(0, T))$ and

$$
\|v\|_{L^{\infty}(\omega \times(0, T))} \leq C\left(1+T^{N-1}\right)\left(1+T^{-1}+\|a\|_{\infty}+\|B\|_{\infty}\right)^{2 N+3}\left(\|\widetilde{w}\|_{Y}+\|\chi\|_{Y}\right) .
$$

At this point, notice that for any $f \in L^{2}(Q)$ and any $y^{0} \in L^{2}(\Omega)$ the solution $y$ to the linear system

$$
\begin{cases}y_{t}-\Delta y+a(x, t) y+B(x, t) \cdot \nabla y=f & \text { in } Q, \\ \frac{\partial y}{\partial n}+\beta(x, t) y=0 & \text { on } \Sigma, \\ y(x, 0)=y^{0}(x) & \text { in } \Omega\end{cases}
$$

satisfies

$$
\|y\|_{Y} \leq \mathrm{e}^{C T\left(1+\|a\|_{\infty}+\|B\|_{\infty}^{2}+\|\beta\|_{\infty}^{2}\right)}\left(\|f\|_{L^{2}(Q)}+\left\|y^{0}\right\|_{L^{2}(\Omega)}\right) .
$$

For a detailed proof, see for example proposition 1 in [10].

This can be used to estimate $\|\widetilde{w}\|_{Y}$ and $\|\chi\|_{Y}$ in terms of $\|\widetilde{v}\|_{L^{2}(\omega \times(0, T))}$ and $\left\|y^{0}\right\|_{L^{2}(\Omega)}$. In view of (17), we see that

$$
\|v\|_{L^{\infty}(\omega \times(0, T))} \leq L\left(\|\widetilde{v}\|_{L^{2}\left(\omega^{\prime \prime} \times(0, T)\right)}+\left\|y^{0}\right\|_{L^{2}(\Omega)}\right),
$$

where

$$
L=C T^{-1}\left(1+T^{N-1}\right)\left(1+T^{-1}+\|a\|_{\infty}+\|B\|_{\infty}\right)^{2 N+3} \exp \left\{C T\left(1+\|a\|_{\infty}+\|B\|_{\infty}^{2}+\|\beta\|_{\infty}^{2}\right)\right\} .
$$

Combining this estimate and (13), we finally obtain that

$$
\|v\|_{L^{\infty}(\omega \times(0, T))} \leq \mathrm{e}^{C K\left(T,\|a\|_{\infty},\|B\|_{\infty},\|\beta\|_{\infty}\right)}\left\|y^{0}\right\|_{L^{2}(\Omega)},
$$

where $K$ is given by (12).

This ends the proof of Proposition 1.

\section{Controllability of the nOnlinear System}

In this section we will prove Theorem 1 . The following auxiliary result will be needed:

Proposition 2. Let us assume that, in (18), we have $f \in L^{\infty}(Q)$ and $y^{0} \in L^{\infty}(\Omega)$. Let us also assume that the coefficients $a, B$ and $\beta$ satisfy (3). Then $y \in L^{\infty}(Q)$ and

$$
\|y\|_{\infty} \leq \mathrm{e}^{C T\left(1+\|a\|_{\infty}+\|B\|_{\infty}^{2}+\|\beta\|_{\infty}^{2}\right)}\left(\left\|y^{0}\right\|_{\infty}+\|f\|_{\infty}\right) .
$$

for some $C=C(\Omega)$.

Proof. We will consider two different situations:

Case 1. We will first assume that $a \geq 1$ and $\beta \geq 0$ and we will establish (21) in this case. In fact, we will show that, under these assumptions,

$$
\|y\|_{\infty} \leq\left\|y^{0}\right\|_{\infty}+\|f\|_{\infty} .
$$

To this end, let us introduce the system

$$
\begin{cases}z_{t}-\Delta z+a(x, t) z+B(x, t) \cdot \nabla z=h & \text { in } Q, \\ \frac{\partial z}{\partial n}+\beta(x, t) z=k & \text { on } \Sigma, \\ z(x, 0)=z^{0}(x) & \text { in } \Omega,\end{cases}
$$


where $h \in L^{\infty}(Q), k \in L^{\infty}(\Sigma)$ and $z^{0} \in L^{\infty}(\Omega)$ and let us show that, if $h, z^{0}$ and $k$ are nonnegative, then this is also the case for $z$.

Indeed, by multiplying the equation satisfied by $z$ by $z_{-}(\cdot, t)$ (the negative part of $z(\cdot, t)$ ) for each $t \in(0, T)$ and integrating in $\Omega$, after several simplifications, we find:

$$
\begin{aligned}
\frac{1}{2} \frac{\mathrm{d}}{\mathrm{d} t} & \int_{\Omega}\left|z_{-}(x, t)\right|^{2} \mathrm{~d} x+\int_{\Omega}\left|\nabla z_{-}(x, t)\right|^{2} \mathrm{~d} x \\
& +\int_{\partial \Omega} \beta(x, t)\left|z_{-}(x, t)\right|^{2} \mathrm{~d} \sigma+\int_{\partial \Omega} k(x, t) z_{-}(x, t) \mathrm{d} \sigma+\int_{\Omega} a(x, t)\left|z_{-}(x, t)\right|^{2} \mathrm{~d} x= \\
& -\int_{\Omega} h(x, t) z_{-}(x, t) \mathrm{d} x-\int_{\Omega} B(x, t) \cdot \nabla z_{-}(x, t) z_{-}(x, t) \mathrm{d} x .
\end{aligned}
$$

From this identity, in view of the positiveness of $a, h, \beta$ and $k$, we easily deduce that

$$
\frac{\mathrm{d}}{\mathrm{d} t} \int_{\Omega}\left|z_{-}(x, t)\right|^{2} \mathrm{~d} x \leq\|B\|_{\infty}^{2} \int_{\Omega}\left|z_{-}(x, t)\right|^{2} \mathrm{~d} x,
$$

whence $z \geq 0$ in $Q$.

Now, let $M>0$ be a large constant (to be chosen below). The function $z=M-y$ satisfies

$$
\begin{cases}z_{t}-\Delta z+a(x, t) z+B(x, t) \cdot \nabla z=a(x, t) M-f & \text { in } Q \\ \frac{\partial z}{\partial n}+\beta(x, t) z=\beta(x, t) M & \text { on } \Sigma \\ z(x, 0)=M-y^{0}(x) & \text { in } \Omega .\end{cases}
$$

Therefore, if we take

$$
M \geq \max \left\{\|f\|_{L^{\infty}(Q)},\left\|y^{0}\right\|_{L^{\infty}(\Omega)}\right\},
$$

we can apply the previous argument and deduce that $y \leq M$. In a similar way, one can deduce that $y \geq-M$ and, consequently, $|y| \leq M$. This proves that whenever $a \geq 1$ and $\beta \geq 0$, the estimate (22) holds.

Case 2. We will now prove (21) for general $L^{\infty}$ coefficients $a$ and $\beta$.

Let $\gamma \in C^{2}(\bar{\Omega})$ be a function satisfying

$$
\begin{gathered}
\gamma \geq 0 \text { in } \Omega, \quad \frac{\partial \gamma}{\partial n} \leq-\|\beta\|_{\infty} \text { on } \partial \Omega, \quad\|\gamma\|_{\infty} \leq 1, \\
\|\nabla \gamma\|_{\infty} \leq C\|\beta\|_{\infty}, \quad\left\|D^{2} \gamma\right\|_{\infty} \leq C\|\beta\|_{\infty}^{2} .
\end{gathered}
$$

We give here a sketch of the proof of the existence of such a function $\gamma$. To this end, let $\delta>0$ be a parameter (depending on $\Omega$ ) such that

$$
x \in \Omega_{\delta} \mapsto \operatorname{dist}(x, \partial \Omega)
$$

is $C^{2}$, with $\Omega_{\delta}=\{x \in \Omega: \operatorname{dist}(x, \partial \Omega)<\delta\}$. We distinguish two cases.

Let us first assume that $\|\beta\|_{\infty} \geq 1 / \delta$. Then we take $\gamma(x) \equiv 1$ in $\Omega \backslash \Omega_{\delta}, \gamma(x)=\|\beta\|_{\infty} \operatorname{dist}(x, \partial \Omega)$ in $\Omega_{\varepsilon}$ with $\varepsilon=1 /\left(2\|\beta\|_{\infty}\right)$ and a regularization of $\gamma$ in $\Omega_{\delta} \backslash \Omega_{\varepsilon}$. This gives the desired properties for $\gamma$.

On the other hand, if $\|\beta\|_{\infty}<1 / \delta$, we take $\gamma(x)=\delta\|\beta\|_{\infty}$ in $\Omega \backslash \Omega_{\delta}, \gamma(x)=\|\beta\|_{\infty} \operatorname{dist}(x, \partial \Omega)$ in $\Omega_{\delta / 2}$ and a regularization in $\Omega_{\delta} \backslash \Omega_{\delta / 2}$. This also provides a desired function in this case. 
Let us now set $\widehat{y}=\mathrm{e}^{\gamma(x)} y$. Then $\widehat{y}$ satisfies

$$
\begin{cases}\widehat{y}_{t}-\Delta \widehat{y}+\widehat{a}(x, t) \widehat{y}+\widehat{B}(x, t) \cdot \nabla \widehat{y}=\mathrm{e}^{\gamma(x)} f & \text { in } Q, \\ \frac{\partial \widehat{y}}{\partial n}+\widehat{\beta}(x, t) \widehat{y}=0 & \text { on } \Sigma, \\ \widehat{y}(x, 0)=\mathrm{e}^{\gamma(x)} y^{0}(x) & \text { in } \Omega,\end{cases}
$$

where

$$
\begin{gathered}
\widehat{a}=a+\Delta \gamma-|\nabla \gamma|^{2}-B \cdot \nabla \gamma, \\
\widehat{B}=B+2 \nabla \gamma, \quad \widehat{\beta}=\beta-\frac{\partial \gamma}{\partial n} \geq 0 \text { on } \Sigma .
\end{gathered}
$$

Notice that, from the inequalities (23) satisfied by $\gamma$, we know that

$$
\left.|a+\Delta \gamma-| \nabla \gamma\right|^{2}-B \cdot \nabla \gamma \mid \leq C_{1}\left(\|a\|_{\infty}+\|B\|_{\infty}^{2}+\|\beta\|_{\infty}^{2}\right) \text { in } Q
$$

for some $C_{1}>0$.

Now, let us set

$$
\widetilde{y}=\mathrm{e}^{-\left(C_{1}\left(\|a\|_{\infty}+\|B\|_{\infty}^{2}+\|\beta\|_{\infty}^{2}\right)+1\right) t} \widehat{y}
$$

Then $\widetilde{y}$ satisfies

$$
\begin{cases}\widetilde{y}_{t}-\Delta \widetilde{y}+\widetilde{a}(x, t) \widetilde{y}+(B(x, t)+2 \nabla \gamma(x)) \cdot \nabla \widetilde{y}=\widetilde{f} & \text { in } Q, \\ \frac{\partial \widetilde{y}}{\partial n}+\widetilde{\beta}(x, t) \widetilde{y}=0 & \text { on } \Sigma, \\ \widetilde{y}(x, 0)=\mathrm{e}^{\gamma(x)} y^{0}(x) & \text { in } \Omega,\end{cases}
$$

where

$$
\begin{gathered}
\widetilde{a}=a+\Delta \gamma-|\nabla \gamma|^{2}-B \cdot \nabla \gamma+C_{1}\left(\|a\|_{\infty}+\|B\|_{\infty}^{2}+\|\beta\|_{\infty}^{2}\right)+1 \\
\widetilde{f}=\mathrm{e}^{-\left(C_{1}\left(\|a\|_{\infty}+\|B\|_{\infty}^{2}+\|\beta\|_{\infty}^{2}\right)+1\right) t+\gamma(x)} f
\end{gathered}
$$

and

$$
\widetilde{\beta}=\widehat{\beta} \text {. }
$$

Since $\widetilde{a} \geq 1$ and $\widetilde{\beta} \geq 0$, we can apply case 1 to $\widetilde{y}$. This provides the estimates

$$
\|y\|_{\infty} \leq\|\widehat{y}\|_{\infty} \leq \mathrm{e}^{C T\left(1+\|a\|_{\infty}+\|B\|_{\infty}^{2}+\|\beta\|_{\infty}^{2}\right)}\left(\left\|y^{0}\right\|_{\infty}+\|f\|_{\infty}\right)
$$

whence we deduce (21).

Let us now start with the proof of Theorem 1. Let $y^{0} \in L^{\infty}(\Omega)$ and $\bar{y}$ be given and assume that $\bar{y}$ satisfies (6) and (4) in the weak sense. Let us consider the nonlinear system

$$
\begin{cases}w_{t}-\Delta w+F_{1}(w, \nabla w ; x, t) w+F_{2}(\nabla w ; x, t) \cdot \nabla w=v 1_{\omega} & \text { in } Q \\ \frac{\partial w}{\partial n}+F_{3}(w ; x, t) w=0 & \text { on } \Sigma \\ w(x, 0)=y^{0}(x)-\bar{y}(x, 0) & \text { in } \Omega\end{cases}
$$

where we have used the notation

$$
\begin{gathered}
F_{1}(s, p ; x, t)=\frac{F(\bar{y}(x, t)+s, \nabla \bar{y}(x, t)+p)-F(\bar{y}(x, t), \nabla \bar{y}(x, t)+p)}{s}, \\
F_{2}=\left(F_{21}, \ldots, F_{2 N}\right), \quad F_{2 j}(p ; x, t)=\int_{0}^{1} \frac{\partial F}{\partial p_{j}}(\bar{y}(x, t), \nabla \bar{y}(x, t)+\lambda p) \mathrm{d} \lambda
\end{gathered}
$$


and

$$
F_{3}(s ; x, t)=\frac{f(\bar{y}(x, t)+s)-f(\bar{y}(x, t))}{s}
$$

for $s \in \mathbf{R}$ and $p \in \mathbf{R}^{N}$.

We will prove that there exist a control $v \in L^{\infty}(\omega \times(0, T))$ and an associated solution to (26) such that

$$
w(x, T)=0 \text { in } \Omega .
$$

With this control and the state $y=w+\bar{y}$, we will have solved the exact controllability problem for (1) and we will have thus proved Theorem 1.

We will first assume that the functions $F$ and $f$ are continuously differentiable. Then, by a density argument, we will be able to prove the result in the general case.

\subsection{The case in which $F$ and $f$ are $C^{1}$}

The idea of the proof is well known: we introduce an appropriate (set-valued) fixed point mapping and we check that it possesses at least one fixed point; this will be a solution to the null controllability problem associated to (26).

Let $R>0$ be given and let us introduce the following function:

$$
M_{R}(s)= \begin{cases}-R & \text { if } s<-R \\ s & \text { if }-R \leq s \leq R \\ R & \text { if } s>R\end{cases}
$$

Let us denote by $Z$ the Hilbert space $Z=L^{2}\left(0, T ; H^{1}(\Omega)\right)$ and let us set for each $R>0$ and each $z \in Z$

$$
\begin{gathered}
a_{R, z}(x, t)=F_{1}\left(M_{R}(z(x, t)), \nabla z(x, t) ; x, t\right), \\
B_{z}(x, t)=F_{2}(\nabla z(x, t) ; x, t)
\end{gathered}
$$

and

$$
\beta_{R, z}(x, t)=F_{3}\left(M_{R}(z(x, t)) ; x, t\right) .
$$

Consider the linear null controllability problem

$$
\begin{cases}w_{t}-\Delta w+a_{R, z}(x, t) w+B_{z}(x, t) \cdot \nabla w=v 1_{\omega} & \text { in } Q \\ \frac{\partial w}{\partial n}+\beta_{R, z}(x, t) w=0 & \text { on } \Sigma \\ w(x, 0)=y^{0}(x)-\bar{y}(x, 0) & \text { in } \Omega\end{cases}
$$

together with (30).

Let us introduce the function $w^{0}$, with $w^{0}(x)=y^{0}(x)-\bar{y}(x, 0)$ for all $x \in \Omega$. From (6), (8) and the fact that $f \in C^{1}(\mathbf{R})$, we have

$$
a_{R, z} \in L^{\infty}(Q), \quad B_{z} \in L^{\infty}(Q)^{N}, \quad \beta_{R, z} \in L^{\infty}(\Sigma)
$$

Consequently, in view of Proposition 1, (30)-(31) can be solved with controls in $L^{\infty}(\omega \times(0, T))$.

We are now going to select a particular solution to (30)-(31) constructed as in [11]. To do this, we first set $T_{R}=\min \left\{T, a_{R}^{-1 / 3}\right\}>0$, where

$$
a_{R}=\sup _{|s| \leq R, p \in \mathbf{R}^{N}} \operatorname{esssup}_{(x, t) \in Q}\left|F_{1}(s, p ; x, t)\right| .
$$


We can follow the steps of Section 1 and construct a control $v_{R, z} \in L^{\infty}\left(\omega \times\left(0, T_{R}\right)\right)$ such that the solution $w_{R, z}$ to $(31)$ in $\Omega \times\left(0, T_{R}\right)$ verifies

$$
w_{R, z}\left(x, T_{R}\right)=0 \text { in } \Omega .
$$

The estimates we have been able to establish in propositions 1 and 2 written for $v_{R, z}$ and $w_{R, z}$ with final time $T_{R}$ will now give

and

$$
\begin{aligned}
& \left\|v_{R, z}\right\|_{L^{\infty}\left(\omega \times\left(0, T_{R}\right)\right)} \leq C_{R}\left\|w^{0}\right\|_{L^{2}(\Omega)}, \\
& \left\|w_{R, z}\right\|_{L^{2}\left(0, T_{R} ; H^{1}(\Omega)\right)} \leq C_{R}\left\|w^{0}\right\|_{L^{2}(\Omega)}
\end{aligned}
$$

$$
\left\|w_{R, z}\right\|_{L^{\infty}\left(\Omega \times\left(0, T_{R}\right)\right)} \leq C_{R}\left\|w^{0}\right\|_{L^{\infty}(\Omega)}
$$

where

and

$$
\begin{gathered}
C_{R}=\exp \left\{C(\Omega, \omega, T)\left(1+a_{R}^{2 / 3}+\bar{B}^{2}+\beta_{R}^{2}\right)\right\}, \\
\bar{B}=\sup _{p \in \mathbf{R}^{N}} \underset{(x, t) \in Q}{\operatorname{ess} \sup }\left|F_{2}(p ; x, t)\right|
\end{gathered}
$$

$$
\beta_{R}=\sup _{|s| \leq R} \operatorname{ess}_{(x, t) \in \Sigma}\left|F_{3}(s ; x, t)\right| .
$$

In fact, the estimates obtained in the previous section imply (32)-(34) with $C_{R}$ replaced by $C_{R}(z)$, where

$$
\begin{aligned}
C_{R}(z)= & \exp \left\{C ( \Omega , \omega ) \left(1+T_{R}^{-1}+T_{R}+\left\|a_{R, z}\right\|_{\infty}^{2 / 3}+\left\|B_{z}\right\|_{\infty}^{2}\right.\right. \\
& \left.\left.+\left\|\beta_{R, z}\right\|_{\infty}^{2}+T_{R}\left(\left\|a_{R, z}\right\|_{\infty}+\left\|B_{z}\right\|_{\infty}^{2}+\left\|\beta_{R, z}\right\|_{\infty}^{2}\right)\right)\right\} ;
\end{aligned}
$$

but taking into account the definitions of $T_{R}, a_{R}, \bar{B}$ and $\beta_{R}$ it is clear that $C_{R}(z) \leq C_{R}$ for all $z \in Z$.

At this moment, we can extend by zero the functions $v_{R, z}$ and $w_{R, z}$ for $t \in\left(T_{R}, T\right)$. In this way, we will have built a control $v_{R, z}$ and an associated state $w_{R, z}$ satisfying (30)-(31) and

and

$$
\begin{gathered}
\left\|v_{R, z}\right\|_{L^{\infty}(\omega \times(0, T))} \leq C_{R}\left\|w^{0}\right\|_{L^{2}(\Omega)}, \\
\left\|w_{R, z}\right\|_{Z} \leq C_{R}\left\|w^{0}\right\|_{L^{2}(\Omega)}
\end{gathered}
$$

We will now introduce a set-valued mapping leading to the solution to our controllability problem.

We first consider the set of admissible controls $A_{R}(z)$. By definition, this is the set of controls $v_{R, z} \in$ $L^{\infty}(\omega \times(0, T))$ which lead the solution to (31) to zero at time $T$ and satisfy (35). Then, for each $z \in Z$, we denote by $\Lambda_{R}(z)$ the set of states $w_{R, z}$ associated to the controls $v_{R, z} \in A_{R}(z)$ furthermore satisfying (36) and (37). In view of the arguments above, $\Lambda_{R}(z)$ is a nonempty subset of $Z$.

The plan of the rest of the proof is the following:

- We will first see that, for each $R>0, \Lambda_{R}$ possesses a fixed point $w_{R}$. This will be implied by Kakutani's theorem.

- Then, we will find $R>0$ (large enough) such that $M_{R}\left(w_{R}\right)=w_{R}$. At this level, the use of Proposition 2 will be crucial.

As a consequence, for large $R$, the fixed point $w_{R}$ of $\Lambda_{R}$ will be, together with some $v_{R} \in L^{\infty}(\omega \times(0, T))$, a solution to (30)-(31).

Thus, let us recall Kakutani's fixed point theorem (see, for instance, [3]):

Theorem 2. Let $Z$ be a Banach space and let $\Lambda: Z \mapsto Z$ be a set-valued mapping satisfying the following assumptions:

1. $\Lambda(z)$ is a nonempty closed convex set of $Z$ for every $z \in Z$.

2. There exists a nonempty convex compact set $K \subset Z$ such that $\Lambda(K) \subset K$. 
3. $\Lambda$ is upper-hemicontinuous in $Z$, i.e. for each $\sigma \in Z^{\prime}$ the single-valued mapping

$$
z \mapsto \sup _{y \in \Lambda(z)}\langle\sigma, y\rangle_{Z^{\prime}, Z}
$$

is upper-semicontinuous.

Then $\Lambda$ possesses a fixed point in the set $K$, i.e. there exists $z \in K$ such that $z \in \Lambda(z)$.

Let us check that Kakutani's theorem can be applied to $\Lambda_{R}$.

That $\Lambda_{R}(z)$ is a nonempty closed convex set of $Z$ for every $z \in Z$ is very easy to verify.

Let us prove that $\Lambda_{R}$ maps a compact set into itself. In fact, let us see that $\Lambda_{R}$ maps the whole space $Z$ into a fixed convex compact set $K_{R}$.

Our argument will be the following: we choose an arbitrary sequence $\left\{z_{n}\right\}$ in $Z$ and a sequence $\left\{w_{n}\right\}$ with $w_{n} \in \Lambda_{R}\left(z_{n}\right)$ for all $n$ and we prove that $\left\{w_{n}\right\}$ possesses a strongly convergent subsequence.

Thus, let the sequences $\left\{z_{n}\right\}$ and $\left\{w_{n}\right\}$ be given. From (35)-(37), the equations satisfied by the functions $w_{n}$ and the fact that $\left\|a_{R, z_{n}}\right\|_{\infty} \leq a_{R},\left\|B_{z_{n}}\right\|_{\infty} \leq \bar{B}$ and $\left\|\beta_{R, z_{n}}\right\|_{\infty} \leq \beta_{R}$ for all $n \geq 1$, we deduce the existence of subsequences $\left\{w_{n^{\prime}}\right\}$ and $\left\{v_{n^{\prime}}\right\}$ such that

$$
\begin{gathered}
w_{n^{\prime}} \rightarrow w \quad \text { weakly in } Z \\
w_{n^{\prime}, t} \rightarrow w_{t} \quad \text { weakly in } L^{2}\left(0, T ; H^{-1}(\Omega)\right)
\end{gathered}
$$

and

$$
v_{n^{\prime}} \rightarrow v \quad \text { weakly-* in } L^{\infty}(Q)
$$

as $n^{\prime} \rightarrow \infty$. We can also assume that the coefficients associated to $z_{n^{\prime}}$ converge weakly-* in $L^{\infty}(Q)$ and $L^{\infty}(\Sigma)$. Thus, we can pass to the limit in the weak formulations satisfied by $w_{n^{\prime}}$ and deduce that $w$ and $v$ satisfy

$$
\begin{cases}w_{t}-\Delta w+a(x, t) w+\theta(x, t)=v 1_{\omega} & \text { in } Q \\ \frac{\partial w}{\partial n}+\beta(x, t) w=0 & \text { on } \Sigma \\ w(x, 0)=w^{0}(x) & \text { in } \Omega\end{cases}
$$

for some $a \in L^{\infty}(Q)$ and $\beta \in L^{\infty}(\Sigma)$, where $\theta \in L^{2}(Q)$ is the weak limit of $B_{z_{n^{\prime}}} \cdot \nabla w_{n^{\prime}}$ in $L^{2}(Q)$.

After substraction of the equations satisfied by the functions $w_{n^{\prime}}$ and $w$, we find that

$$
\begin{cases}\left(w_{n^{\prime}}-w\right)_{t}-\Delta\left(w_{n^{\prime}}-w\right)=a(x, t) w-a_{R, z_{n^{\prime}}}(x, t) w_{n^{\prime}} & \\ +\theta(x, t)-B_{z_{n^{\prime}}}(x, t) \cdot \nabla w_{n^{\prime}}+\left(v_{n^{\prime}}-v\right) 1_{\omega} & \text { in } Q \\ \frac{\partial\left(w_{n^{\prime}}-w\right)}{\partial n}+\beta_{R, z_{n^{\prime}}}(x, t) w_{n^{\prime}}-\beta(x, t) w=0 & \text { on } \Sigma \\ \left(w_{n^{\prime}}-w\right)(x, 0)=0 & \text { in } \Omega .\end{cases}
$$

Consequently,

$$
\begin{array}{r}
\frac{1}{2} \int_{\Omega}\left|\left(w_{n^{\prime}}-w\right)(x, T)\right|^{2} \mathrm{~d} x+\int_{0}^{T} \int_{\Omega}\left|\nabla\left(w_{n^{\prime}}-w\right)(x, s)\right|^{2} \mathrm{~d} x \mathrm{~d} s=\int_{0}^{T} \int_{\partial \Omega}\left(\beta w-\beta_{R, z_{n^{\prime}}} w_{n^{\prime}}\right)\left(w_{n^{\prime}}-w\right)(x, s) \mathrm{d} \sigma \mathrm{d} s \\
+\int_{0}^{T} \int_{\Omega}\left(a w-a_{R, z_{n^{\prime}}} w_{n^{\prime}}\right)\left(w_{n^{\prime}}-w\right)(x, s) \mathrm{d} x \mathrm{~d} s+\int_{0}^{T} \int_{\Omega}\left(\theta-B_{z_{n^{\prime}}} \cdot \nabla w_{n^{\prime}}\right)\left(w_{n^{\prime}}-w\right)(x, s) \mathrm{d} x \mathrm{~d} s \\
+\int_{0}^{T} \int_{\omega}\left(v_{n^{\prime}}-v\right)\left(w_{n^{\prime}}-w\right)(x, s) \mathrm{d} x \mathrm{~d} s . \quad(39)
\end{array}
$$


We are now going to check that all the terms in the right hand side of this last equality tends to zero. Among other things, this will imply that

$$
w_{n^{\prime}} \rightarrow w \quad \text { strongly in } Z \text {. }
$$

- The first term in the right hand side converges to zero, since

$$
w_{n^{\prime}} \rightarrow w \quad \text { strongly in } L^{2}(\Sigma)
$$

and consequently

$$
\beta_{R, z_{n^{\prime}}} w_{n^{\prime}} \rightarrow \beta w \quad \text { weakly in } L^{2}(\Sigma) .
$$

Indeed, the strong convergence of $w_{n^{\prime}}$ is an immediate consequence of the compact embedding of the space

$$
\left\{z \in L^{2}\left(0, T ; H^{1}(\Omega)\right): z_{t} \in L^{2}\left(0, T ; H^{-1}(\Omega)\right)\right\}
$$

in $L^{2}\left(0, T ; H^{s}(\Omega)\right)$ for all $s \in(1 / 2,1)$ and the fact that the lateral trace operator is well defined, linear and continuous from $L^{2}\left(0, T ; H^{s}(\Omega)\right)$ into $L^{2}(\Sigma)$.

- The convergence of the other three terms in the right hand side is a consequence of the weak convergence in $L^{2}(Q)$ of $a_{R, z_{n^{\prime}}} w_{n^{\prime}}$ and $B_{z_{n^{\prime}}} \cdot \nabla w_{n^{\prime}}$, the weak convergence in $L^{2}(\omega \times(0, T))$ of $v_{n^{\prime}}$ and the strong convergence of $w_{n^{\prime}}$ in $L^{2}(Q)$.

We have thus seen that $\left\{w_{n}\right\}$ possesses a strongly convergent subsequence and, consequently, $\Lambda_{R}$ maps the space $Z$ into a fixed compact set.

It remains to check that $\Lambda_{R}$ is upper-hemicontinuous. Thus, assume that $\sigma \in Z^{\prime}$ and let a sequence $\left\{z_{n}\right\}$ be given, with $z_{n} \rightarrow z$ strongly in $Z$. We must prove that

$$
\varlimsup_{n \rightarrow+\infty} \sup _{w \in \Lambda_{R}\left(z_{n}\right)}\langle\sigma, w\rangle_{Z^{\prime}, Z} \leq \sup _{w \in \Lambda_{R}(z)}\langle\sigma, w\rangle_{Z^{\prime}, Z} .
$$

Let $\left\{z_{n^{\prime}}\right\}$ be a subsequence of $\left\{z_{n}\right\}$ such that

$$
\varlimsup_{n \rightarrow+\infty} \sup _{w \in \Lambda_{R}\left(z_{n}\right)}\langle\sigma, w\rangle_{Z^{\prime}, Z}=\lim _{n^{\prime} \rightarrow+\infty} \sup _{w \in \Lambda_{R}\left(z_{n^{\prime}}\right)}\langle\sigma, w\rangle_{Z^{\prime}, Z} .
$$

Since each $\Lambda_{R}\left(z_{n^{\prime}}\right)$ is a compact set of $Z$, for every $n^{\prime}$ we have

$$
\sup _{w \in \Lambda_{R}\left(z_{n^{\prime}}\right)}\langle\sigma, w\rangle_{Z^{\prime}, Z}=\left\langle\sigma, w_{n^{\prime}}\right\rangle_{Z^{\prime}, Z}
$$

for some $w_{n^{\prime}} \in \Lambda_{R}\left(z_{n^{\prime}}\right)$. On the other hand, since all the states $w_{n^{\prime}}$ belong to the same compact set $K_{R}$, at least for a new subsequence (again indexed by $n^{\prime}$ ), we must have (40). We will now prove that $w \in \Lambda_{R}(z)$. This will achieve the proof of the upper hemicontinuity of $\Lambda_{R}$.

Indeed, we can assume that the weak limits of the coefficients associated to $z_{n^{\prime}}$ are $a_{R, z}, B_{z}$ and $\beta_{R, z}$, since $z_{n^{\prime}}$ converges strongly in $Z$ towards $z$ and therefore the coefficients $a_{R, z_{n^{\prime}}}, B_{z_{n^{\prime}}}$ and $\beta_{R, z_{n^{\prime}}}$ converge almost everywhere (observe that we are using here the $C^{1}$ regularity of $F$ and $f$ ).

On the other hand, it can be assumed that the controls $v_{n^{\prime}}$ converge to a function $v$ weakly- $*$ in $L^{\infty}(\omega \times(0, T))$. Then, $w$ solves (31) and $w(T)=0$. Moreover, since inequality (35) is independent of $n, v$ also satisfies (35). Therefore, $v \in A_{R}(z)$. Consequently, it is immediate that $w$ is the solution to (31) associated to the control $v$.

This shows that $w \in \Lambda_{R}(z)$ and, therefore, $\Lambda_{R}$ is upper hemicontinuous.

In view of these arguments, Kakutani's theorem can be applied and we deduce that, for each $R>0, \Lambda_{R}$ possesses at least one fixed point $w_{R}$ that belongs to $Z$ and $L^{\infty}(Q)$.

Our aim is now to find $R>0$ such that

$$
\left\|w_{R}\right\|_{\infty} \leq R
$$

This will be a consequence of the estimates we know for $w_{R}$ and the properties satisfied by the functions $F_{i}$. 
From (37), we obtain

$$
\left\|w_{R}\right\|_{\infty} \leq \mathrm{e}^{C(\Omega, \omega, T)\left(1+a_{R}^{2 / 3}+\bar{B}^{2}+\beta_{R}^{2}\right)}\left\|w^{0}\right\|_{\infty} .
$$

On the other hand, from (8)-(9) it is also clear that, for every $\varepsilon>0$, there exists $C_{\varepsilon}>0$ such that

$$
\begin{gathered}
\left(\underset{(x, t) \in Q}{\operatorname{ess} \sup _{0}}\left|F_{1}(s, p ; x, t)\right|\right)^{2 / 3} \leq \varepsilon \log (1+|s|)+C_{\varepsilon} \quad \forall s \in \mathbf{R}, \forall p \in \mathbf{R}^{N}, \\
\left(\operatorname{esssup}_{(x, t) \in Q}\left|F_{2}(p ; x, t)\right|\right)^{2} \leq C_{\varepsilon} \quad \forall p \in \mathbf{R}^{N}, \\
\left(\operatorname{esssup}_{(x, t) \in \Sigma}\left|F_{1}(s ; x, t)\right|\right)^{2} \leq \varepsilon \log (1+|s|)+C_{\varepsilon} \quad \forall s \in \mathbf{R} .
\end{gathered}
$$

Consequently, it is also true that, for every $\varepsilon>0$, there exists $C_{\varepsilon}>0$ (independent of $R$ ) such that

$$
a_{R}^{2 / 3}+\bar{B}^{2}+\beta_{R}^{2} \leq \varepsilon \log (1+R)+C_{\varepsilon} .
$$

These estimates, together with (41) and the definitions of $a_{R}, B$ and $\beta_{R}$, lead to the following inequality:

$$
\left\|w_{R}\right\|_{\infty} \leq C(\Omega, \omega, T, \varepsilon)(1+R)^{C(\Omega, \omega, T) \varepsilon}\left\|w^{0}\right\|_{\infty} .
$$

Accordingly, taking $\varepsilon>0$ small enough to satisfy $C(\Omega, \omega, T) \varepsilon<1$, we can ensure that, for $R>0$ sufficiently large (depending on $\Omega, \omega, T$ and $\left\|y^{0}-\bar{y}^{0}\right\|_{L^{\infty}(\Omega)}$ ), one has

$$
\left\|w_{R}\right\|_{\infty} \leq R .
$$

This ends the proof of theorem 1 when $F$ and $f$ are $C^{1}$ functions.

Remark 3. One could think about considering a nonlinearity $F$ also having a slightly superlinear growth in the $p$ variables, as in [6]; this would mean a condition similar to (7) but in the $p$ variables instead of the $s$ variable. However, our arguments do not work in this case. Indeed, we would need uniform estimates of the states in $L^{\infty}\left(0, T ; W^{1, \infty}(\Omega)\right)$ and this is not possible if the coefficients $\beta_{R, z}$ only belong to $L^{\infty}(\Sigma)$ (further regularity with respect to the time variable would be needed).

\subsection{The general case}

We will now assume that $f$ and $F$ are locally Lipschitz-continuous functions satisfying (7)-(9).

Let us introduce the functions $\rho_{1} \in C_{c}^{\infty}\left(\mathbf{R} \times \mathbf{R}^{N}\right), \rho_{2} \in C_{c}^{\infty}\left(\mathbf{R}^{N}\right)$ and $\rho_{3} \in C_{c}^{\infty}(\mathbf{R})$, with $\rho_{j} \geq 0$, supp $\rho_{1} \subset$ $\bar{B}((0,0) ; 1)$, supp $\rho_{2} \subset \bar{B}(0 ; 1)$, supp $\rho_{3} \subset[-1,1]$ and

$$
\iint_{\mathbf{R} \times \mathbf{R}^{N}} \rho_{1}(s, p) \mathrm{d} s \mathrm{~d} p=\int_{\mathbf{R}^{N}} \rho_{2}(p) \mathrm{d} p=\int_{\mathbf{R}} \rho_{3}(s) \mathrm{d} s=1 .
$$

Let us consider, for each $n \geq 1$, the associated mollifiers

$$
\rho_{1, n}(s, p)=n^{N+1} \rho_{1}(n s, n p), \quad \rho_{2, n}(p)=n^{N} \rho_{2}(n p) \quad \forall(s, p) \in \mathbf{R} \times \mathbf{R}^{N}
$$

and

$$
\rho_{3, n}(s)=n \rho_{3}(n s) \quad \forall s \in \mathbf{R}
$$


and the regularized functions

$$
F_{i, n}=\rho_{i, n} * F_{i} \quad(i=1,2,3)
$$

(the functions $F_{1}, F_{2}$ and $F_{3}$ were defined in (27)-(29)).

These functions satisfy the following:

- For each $n \geq 1, F_{1 n}: \mathbf{R} \times \mathbf{R}^{N} \times Q \mapsto \mathbf{R}, F_{2 n}: \mathbf{R}^{N} \times Q \mapsto \mathbf{R}^{N}$ and $F_{3 n}: \mathbf{R} \times \Sigma \mapsto \mathbf{R}$ are Caratheodory functions (respectively continuous in $(s, p), p$ and $s$ and measurable in $(x, t)$ ).

- If we set

$$
F_{n}(s, p ; x, t)=F_{1, n}(s, p ; x, t) s+F_{2, n}(p ; x, t) \cdot p
$$

and

$$
f_{n}(s ; x, t)=F_{3, n}(s ; x, t) s,
$$

then the asymptotic properties (8) and (42) remain true uniformly in $n$. In other words, for any $L>0$, there exists $M>0$ (independent of $n$ ) such that

$$
\left\{\begin{array}{l}
\left|F_{n}(s, p)-F_{n}(r, p)\right| \leq M|s-r|, \quad\left|F_{n}(s, p)-F_{n}(s, q)\right| \leq M|p-q| \\
\forall(s, r, p, q) \in[-L, L]^{2} \times \mathbf{R}^{N} \times \mathbf{R}^{N}
\end{array}\right.
$$

Moreover, for each $\varepsilon>0$, there exists $C_{\varepsilon}>0$ such that

$$
\left\{\begin{array}{l}
\left(\operatorname{essup}_{(x, t) \in Q}\left|F_{1 n}(s, p ; x, t)\right|\right)^{2 / 3} \leq \varepsilon \log (1+|s|)+C_{\varepsilon} \quad \forall s \in \mathbf{R}, \forall p \in \mathbf{R}^{N}, \\
\left(\operatorname{essup}_{(x, t) \in Q}\left|F_{2 n}(p ; x, t)\right|\right)^{2} \leq C_{\varepsilon} \quad \forall p \in \mathbf{R}^{N}, \\
\left(\operatorname{essup}_{(x, t) \in \Sigma}\left|F_{3 n}(s ; x, t)\right|\right)^{2} \leq \varepsilon \log (1+|s|)+C_{\varepsilon} \quad \forall s \in \mathbf{R},
\end{array}\right.
$$

for each $n \geq 1$.

- From the definitions of $F_{n}$ and $f_{n}$, we also have that

$$
F_{n}\left(z_{n}, \nabla z_{n} ; \cdot\right) \rightarrow F_{1}(z, \nabla z ; \cdot) z+F_{2}(\nabla z ; \cdot) \cdot \nabla z \quad \text { weakly in } L^{2}(Q)
$$

and

$$
f_{n}\left(z_{n} ; \cdot\right) \rightarrow F_{3}(z ; \cdot) z \quad \text { weakly-* in } L^{\infty}(\Sigma)
$$

whenever

$$
z_{n} \rightarrow z \quad \text { weakly-* in } L^{\infty}(Q) \text { and strongly in } L^{2}\left(0, T ; H^{1}(\Omega)\right) .
$$

As a consequence, we can argue as in Section 3.1 and deduce that, for each $n$, there exists a control $v_{n} \in$ $L^{\infty}(\omega \times(0, T))$ such that

$$
\begin{cases}w_{n, t}-\Delta w_{n}+F_{n}\left(w_{n}, \nabla w_{n} ; x, t\right)=v_{n} 1_{\omega} & \text { in } Q \\ \frac{\partial w_{n}}{\partial n}+f_{n}\left(w_{n} ; x, t\right)=0 & \text { on } \Sigma \\ w_{n}(x, 0)=w^{0}(x) & \text { in } \Omega\end{cases}
$$


and

$$
w_{n}(x, T)=0 \text { in } \Omega .
$$

In view of the properties satisfied by the functions $F_{i n}$, the estimates we have established in Section 3.1 are independent of $n$. Accordingly, at least for a subsequence, we also have

$$
\begin{gathered}
v_{n} \rightarrow v \quad \text { weakly-* in } L^{\infty}(\omega \times(0, T)), \\
w_{n, t} \rightarrow w_{t} \quad \text { weakly in } L^{2}\left(0, T ; H^{-1}(\Omega)\right)
\end{gathered}
$$

and

$$
w_{n} \rightarrow w \quad \text { weakly-* in } L^{\infty}(Q) \text { and strongly in } L^{2}\left(0, T ; H^{1}(\Omega)\right) .
$$

Thus, we can pass to the limit in (43) and find a control $v \in L^{\infty}(\omega \times(0, T))$ such that the associated solution to (26) satisfies (30).

This ends the proof of Theorem 1.

Remark 4. The proof of theorem 1 can also be achieved by applying another fixed point argument. More precisely, we can first introduce a small parameter $\varepsilon>0$ and find a control $v_{\varepsilon}$ such that the solution of (1) satisfies

$$
\|y(\cdot, T)-\bar{y}(\cdot, T)\|_{L^{2}} \leq \varepsilon
$$

This can be made by previously solving an approximate controllability problem for the linear system (31) with the control of minimal norm in $L^{p}(\omega \times(0, T))$ for $p$ large enough and, then, using Schauder's theorem. Since all the estimates we can establish are uniform in $\varepsilon$, we can pass to the limit as $\varepsilon \rightarrow 0$ and deduce the desired result.

\section{Appendix: Proof of Lemma 1}

Let us introduce $N+1$ open subsets of $\mathcal{O}$ satisfying

$$
\mathcal{O}_{N}=\mathcal{O}^{\prime} \subset \subset \mathcal{O}_{N-1} \subset \subset \ldots \subset \subset \mathcal{O}_{1} \subset \subset \mathcal{O}_{0} \subset \subset \mathcal{O} .
$$

We will also consider subintervals of $(0, T)$ of the form $(\delta /(i+1), T)$ for $0 \leq i \leq N$.

We will strict our considerations to the proof of Lemma 1 in the case where no initial condition is imposed. The result concerning a vanishing initial condition will follow readily from the argument below.

We are first going to see that

$$
y \in L^{\infty}\left(\delta /(N+1), T ; H^{1}\left(\mathcal{O}_{0}\right)\right) \quad \text { and } \quad \Delta y \in L^{2}\left(\delta /(N+1), T ; L^{2}\left(\mathcal{O}_{0}\right)\right),
$$

with an estimate of the associated norms independent of $T$. To this end, let $\xi_{0} \in C_{c}^{2}(\mathcal{O})$ and $\eta_{0} \in C^{1}([0, T])$ be two functions satisfying

$$
\xi_{0}(x)=1 \text { in } \mathcal{O}_{0}, \quad \eta_{0}(t)=1 \text { in }\left[\frac{\delta}{N+1}, T\right], \quad \eta_{0}(0)=0, \quad\left|\eta_{0, t}(t)\right| \leq \frac{C}{\delta} \text { in }(0, T)
$$

(of course, $C$ depends on $N$ ) and let us introduce the function $y_{0}=\eta_{0} \xi_{0} y$. Then

$$
\begin{cases}y_{0, t}-\Delta y_{0}=f_{0} & \text { in } Q, \\ y_{0}=0 & \text { on } \Sigma, \\ y_{0}(x, 0)=0 & \text { in } \Omega,\end{cases}
$$

where

$$
f_{0}=\eta_{0} \xi_{0} f+\eta_{0, t} \xi_{0} y-2 \eta_{0} \nabla \xi_{0} \cdot \nabla y-\eta_{0} \Delta \xi_{0} y-a \eta_{0} \xi_{0} y-\eta_{0} \xi_{0} B \cdot \nabla y-\eta_{0}\left(B \cdot \nabla \xi_{0}\right) y
$$


We have $f_{0} \in L^{2}(Q)$. Consequently, $\Delta y_{0} \in L^{2}(Q), y_{0} \in C^{0}\left([0, T] ; H_{0}^{1}(\Omega)\right)$ and appropriate estimates are satisfied. Indeed, by multiplying the equation satisfied by $y_{0}$ by $-\Delta y_{0}$ and integrating with respect to $x$ in $\Omega$, we find

Since

$$
\frac{1}{2} \frac{\mathrm{d}}{\mathrm{d} t}\left\|\nabla y_{0}(\cdot, t)\right\|_{L^{2}}^{2}+\int_{\Omega}\left|\Delta y_{0}(x, t)\right|^{2} \mathrm{~d} x=-\int_{\Omega} f_{0}(x, t) \Delta y_{0}(x, t) \mathrm{d} x .
$$

we easily obtain from (45) that

$$
\left\|f_{0}\right\|_{L^{2}} \leq C\left(\|f\|_{L^{2}}+\left(1+\delta^{-1}+\|a\|_{\infty}+\|B\|_{\infty}\right)\|y\|_{Y}\right),
$$

$$
\left\|y_{0}\right\|_{C^{0}\left([0, T] ; H_{0}^{1}(\Omega)\right)}+\left\|\Delta y_{0}\right\|_{L^{2}(Q)} \leq C\left(\|f\|_{L^{2}}+\left(1+\delta^{-1}+\|a\|_{\infty}+\|B\|_{\infty}\right)\|y\|_{Y}\right) .
$$

Clearly, the same estimate holds for

$$
\|y\|_{L^{\infty}\left(\delta /(N+1), T ; H^{1}\left(\mathcal{O}_{0}\right)\right)}+\|\Delta y\|_{L^{2}\left(\mathcal{O}_{0} \times(\delta /(N+1), T)\right)} .
$$

Let us now try to improve the local space regularity properties of $y$. To this end, we will use the following lemma:

Lemma 2. Let us set $p_{0}=2$, let $p_{i}$ be defined by

$$
\frac{1}{p_{i}}=\frac{1}{p_{i-1}}-\frac{1}{2 N}
$$

for $1 \leq i \leq N-1$ and let us set $p_{N}=+\infty$. Let us denote by $X_{i}$ the space

$$
X_{i}=L^{\infty}\left((i+1) \delta /(N+1), T ; W^{1, p_{i}}\left(\mathcal{O}_{i}\right)\right)
$$

for $0 \leq i \leq N$ and suppose that $y \in X_{j-1}$ for some $j$. Then we also have $y \in X_{j}$ and

$$
\|y\|_{X_{j}} \leq C\left(\mathcal{O}^{\prime}\right)\left(T^{1 / 2}\|f\|_{\infty}+D\left(T, \delta,\|a\|_{\infty},\|B\|_{\infty}\right)\|y\|_{X_{j-1}}\right),
$$

where

$$
D\left(T, \delta,\|a\|_{\infty},\|B\|_{\infty}\right)=\left(T^{1 / 4}+T^{1 / 2}\right)\left(1+\delta^{-1}+\|a\|_{\infty}+\|B\|_{\infty}\right) .
$$

Proof of Lemma 2. Let us introduce $\xi_{j} \in C_{c}^{2}\left(\mathcal{O}_{j-1}\right)$ and $\eta_{j} \in C^{1}([0, T])$, with

$$
\begin{gathered}
\xi_{j}(x)=1 \text { in } \mathcal{O}_{j}, \quad \eta_{j}(t)=1 \text { in }[(j+1) \delta /(N+1), T], \\
\eta_{j}(t)=0 \text { in }[0, j \delta /(N+1)], \quad\left|\eta_{j, t}(t)\right| \leq \frac{C}{\delta} \text { in }(0, T)
\end{gathered}
$$

and let us put $y_{j}=\eta_{j} \xi_{j} y$. Then $y_{j}$ satisfies the following:

$$
\begin{cases}y_{j, t}-\Delta y_{j}=f_{j} & \text { in } Q, \\ y_{j}=0 & \text { on } \Sigma, \\ y_{j}(x, 0)=0 & \text { in } \Omega\end{cases}
$$

with

where

$$
f_{j}=f_{j, 1}+f_{j, 2}+f_{j, 3}
$$

$$
\begin{gathered}
f_{j, 1}=\eta_{j} \xi_{j} f, \quad f_{j, 2}=\eta_{j, t} \xi_{j} y-\eta_{j} \Delta \xi_{j} y-a \eta_{j} \xi_{j} y-\eta_{j}\left(B \cdot \nabla \xi_{j}\right) y, \\
f_{j, 3}=-2 \eta_{j} \nabla \xi_{j} \cdot \nabla y-\eta_{j} \xi_{j} B \cdot \nabla y
\end{gathered}
$$


From the fact that the system (48) is linear, we see that $y_{j}$ can be written as the sum of three solutions to similar systems with right hand sides $f_{j, 1}, f_{j, 2}$ and $f_{j, 3}$. Let us respectively denote them by $y_{j, 1}, y_{j, 2}$ and $y_{j, 3}$. We are now going to deduce estimates of $y_{j, k}$ in $X_{j}$ for $1 \leq k \leq 3$.

To this end, we will use the usual representation of $y_{j, k}$ provided by the semigroup $S(t)$ associated to the heat equation with homogeneous Dirichlet conditions, say

$$
y_{j, k}(\cdot, t)=\int_{0}^{t} S(t-s) f_{j, k}(\cdot, s) \mathrm{d} s
$$

for all $t \in(0, T)$.

Since $f \in L^{\infty}(Q)$, we can write

$$
\left\|y_{j, 1}(\cdot, t)\right\|_{W^{1, p_{j}(\Omega)}} \leq C \int_{0}^{t}(t-s)^{-1 / 2}\left\|f_{j, 1}(\cdot, s)\right\|_{L^{p_{j}(\Omega)}} \mathrm{d} s .
$$

Therefore, from Young's inequality we find that $y_{j, 1} \in L^{\infty}\left(0, T ; W^{1, p_{j}}(\Omega)\right)$ and

$$
\begin{aligned}
\left\|y_{j, 1}\right\|_{L^{\infty}\left(0, T ; W^{1, p_{j}}(\Omega)\right)} & \leq C T^{1 / 2}\left\|f_{j, 1}\right\|_{L^{\infty}\left(0, T ; L^{p_{j}}(\Omega)\right)} \\
& \leq C\left(\mathcal{O}^{\prime}\right) T^{1 / 2}\|f\|_{L^{\infty}(\mathcal{O} \times(0, T))}
\end{aligned}
$$

Taking into account that $f_{j, 2} \in L^{\infty}\left(0, T ; L^{p_{j-1}^{*}}(\Omega)\right)$ with

$$
p_{j-1}^{*}= \begin{cases}\infty & \text { if } j>N-1, \\ p & (\operatorname{arbitrary} \text { in }(1,+\infty)) \text { if } j=N-1, \\ \frac{2 N}{N-j-1} & \text { if } j<N-1,\end{cases}
$$

we see that $f_{j, 2}$ is not worse than $f_{j, 1}$ and, again,

$$
\left\|y_{j, 2}(\cdot, t)\right\|_{W^{1, p_{j}}(\Omega)} \leq C \int_{0}^{t}(t-s)^{-1 / 2}\left\|f_{j, 2}(\cdot, s)\right\|_{L^{p_{j}(\Omega)}} \mathrm{d} s
$$

for all $t$. From Young's inequality and the assumption $y \in X_{j-1}$, we also get $y_{j, 2} \in L^{\infty}\left(0, T ; W^{1, p_{j}}(\Omega)\right)$ and

$$
\begin{aligned}
\left\|y_{j, 2}\right\|_{L^{\infty}\left(0, T ; W^{1, p_{j}}(\Omega)\right)} & \leq C T^{1 / 2}\left\|f_{j, 2}\right\|_{L^{\infty}\left(0, T ; L^{p_{j-1}^{*}}(\Omega)\right)} \\
& \leq C\left(\mathcal{O}^{\prime}\right) T^{1 / 2}\left(1+\delta^{-1}+\|a\|_{\infty}\right)\|y\|_{X_{j-1}} .
\end{aligned}
$$

In the definition of $f_{j, 3}$, we find $\nabla y$. Consequently, we can only ensure that $f_{j, 3} \in L^{\infty}\left(0, T ; L^{p_{j-1}}(\Omega)\right)$. Since

$$
-\frac{N}{2}\left(\frac{1}{p_{j-1}}-\frac{1}{p_{j}}\right)-\frac{1}{2}=-\frac{3}{4},
$$

we have

$$
\left\|y_{j, 3}(\cdot, t)\right\|_{W^{1, p_{j}(\Omega)}} \leq C \int_{0}^{t}(t-s)^{-3 / 4}\left\|f_{j, 3}(\cdot, s)\right\|_{L^{p_{j-1}(\Omega)}} \mathrm{d} s
$$

and now Young's inequality gives $y_{j, 3} \in L^{\infty}\left(0, T ; W^{1, p_{j}}(\Omega)\right)$ and

$$
\begin{aligned}
\left\|y_{j, 3}\right\|_{L^{\infty}\left(0, T ; W^{1, p_{j}}(\Omega)\right)} & \leq C T^{1 / 4}\left\|f_{j, 3}\right\|_{L^{\infty}\left(0, T ; L^{p_{j-1}}(\Omega)\right)} \\
& \leq C\left(\mathcal{O}^{\prime}\right) T^{1 / 4}\left(1+\|B\|_{\infty}\right)\|y\|_{X_{j-1}} .
\end{aligned}
$$


Putting the estimates of $\left\|y_{j, k}\right\|_{L^{\infty}\left(0, T ; W^{1, p_{j}}(\Omega)\right)}$ together and taking into account the definitions of $\eta_{j}$ and $\xi_{j}$, we obtain the desired inequality for $\|y\|_{X_{j}}$.

This concludes the proof of Lemma 2.

Since we already had $y \in X_{0}$, we deduce from Lemma 2 that $y \in X_{N}$ and

$$
\|y\|_{X_{N}} \leq C\left(\mathcal{O}^{\prime}\right)\left(T^{1 / 2}\|f\|_{\infty}+D\left(T, \delta,\|a\|_{\infty},\|B\|_{\infty}\right)\|y\|_{X_{N-1}}\right),
$$

where, $D$ is given by (47).

We can apply Lemma 2 subsequently for $j=N, N-1, \ldots, 1$. The estimates we find yield

$$
\|y\|_{X_{N}} \leq C\left(T^{1 / 2}\left(1+D^{N-1}\right)\|f\|_{L^{\infty}(\mathcal{O} \times(0, T))}+D^{N}\|y\|_{X_{0}}\right) .
$$

This, together with (46), yields

$$
\|y\|_{X_{N}} \leq C\left(T^{1 / 2}+T^{N / 2}\right) D\left(T, \delta,\|a\|_{\infty},\|B\|_{\infty}\right)^{N+1}\left(\|f\|_{L^{\infty}(\mathcal{O} \times(0, T))}+\|y\|_{Y}\right),
$$

which is exactly (15).

\section{REFERENCES}

[1] H. Amann, Parabolic evolution equations and nonlinear boundary conditions. J. Diff. Equ. 72 (1988) $201-269$.

[2] J. Arrieta, A. Carvalho and A. Rodríguez-Bernal, Parabolic problems with nonlinear boundary conditions and critical nonlinearities. J. Diff. Equ. 156 (1999) 376-406.

[3] J.P. Aubin, L'analyse non linéaire et ses motivations économiques. Masson, Paris (1984).

[4] O. Bodart, M. González-Burgos and R. Pŕez-García, Insensitizing controls for a semilinear heat equation with a superlinear nonlinearity. C. R. Math. Acad. Sci. Paris 335 (2002) 677-682.

[5] A. Doubova, E. Fernández-Cara and M. González-Burgos, On the controllability of the heat equation with nonlinear boundary Fourier conditions. J. Diff. Equ. 196 (2004) 385-417.

[6] A. Doubova, E. Fernández-Cara, M. González-Burgos and E. Zuazua, On the controllability of parabolic systems with a nonlinear term involving the state and the gradient. SIAM J. Control Optim. 41 (2002) 798-819.

[7] L. Evans, Regularity properties of the heat equation subject to nonlinear boundary constraints. Nonlinear Anal. 1 (1997) 593-602.

[8] C. Fabre, J.P. Puel and E. Zuazua, Approximate controllability of the semilinear heat equation. Proc. Roy. Soc. Edinburgh 125A (1995) 31-61.

[9] L.A. Fernández and E. Zuazua, Approximate controllability for the semi-linear heat equation involving gradient terms. $J$. Optim. Theory Appl. 101 (1999) 307-328.

[10] E. Fernández-Cara, M. González-Burgos, S. Guerrero and J.P. Puel, Null controllability of the heat equation with boundary Fourier conditions: The linear case. ESAIM: COCV 12 442-465.

[11] E. Fernández-Cara and E. Zuazua, Null and approximate controllability for weakly blowing up semilinear heat equations. Ann. Inst. H. Poincaré, Anal. non Linéaire 17 (2000) 583-616.

[12] A. Fursikov and O.Yu. Imanuvilov, Controllability of Evolution Equations. Lecture Notes \#34, Seoul National University, Korea (1996).

[13] I. Lasiecka and R. Triggiani, Exact controllability of semilinear abstract systems with applications to waves and plates boundary control. Appl. Math. Optim. 23 (1991) 109-154.

[14] I. Lasiecka and R. Triggiani, Control Theory for Partial Differential Equations: Continuous and Approximation Theories. Cambridge University Press, Cambridge (2000).

[15] E. Zuazua, Exact boundary controllability for the semilinear wave equation, in Nonlinear Partial Differential Equations and their Applications, Vol. X, H. Brezis and J.L. Lions Eds. Pitman (1991) 357-391.

[16] E. Zuazua, Exact controllability for the semilinear wave equation in one space dimension. Ann. I.H.P., Analyse non Linéaire 10 (1993) 109-129. 